\title{
Effects of high-intensity interval training combined with traditional strength or power training on functionality and physical fitness in healthy older men: A randomized controlled trial
}

\author{
Diana Carolina Müller ${ }^{\mathrm{a}}$, Francesco Pinto Boeno ${ }^{\mathrm{a}}$, Mikel Izquierdo ${ }^{\text {b,c }}$, Per Aagaard ${ }^{\mathrm{d}}$, \\ Juliana Lopes Teodoro ${ }^{a}$, Rafael Grazioli ${ }^{a}$, Giovani Cunha ${ }^{a}$, Rodrigo Ferrari ${ }^{\text {a, }}$, \\ Mikel L. Saez de Asteasu ${ }^{\mathrm{b}}$, Ronei Silveira Pinto ${ }^{\mathrm{a}}$, Eduardo Lusa Cadore ${ }^{\mathrm{a}, *}$ \\ ${ }^{a}$ Exercise Research Laboratory, School of Physical Education, Physiotherapy and Dance, Universidade Federal do Rio Grande do Sul, Brazil \\ ${ }^{\mathrm{b}}$ Navarrabiomed, Complejo Hospitalario de Navarra (CHN)-Universidad Pública de Navarra (UPNA), IDISNA, Pamplona, Navarra, Spain \\ ${ }^{\mathrm{c}}$ CIBER of Frailty and Healthy Aging (CIBERFES), Instituto de Salud Carlos III, Madrid, Spain. \\ ${ }^{\mathrm{d}}$ Department of Sports Science and Clinical Biomechanics, Research Unit for Muscle Physiology and Biomechanics, University of Southern Denmark, Denmark \\ ${ }^{\mathrm{e}}$ Postgraduate Program in Cardiology, Universidade Federal do Rio Grande do Sul, Porto Alegre, RS, Brazil
}

\section{A R T I C L E I N F O}

Section editor: Emanuele Marzetti

\section{Keywords:}

Concurrent training

Muscle power

Functional performance

Endurance performance

Movement economy

Aging

\begin{abstract}
A B S T R A C T
Concurrent training (CT) is an efficient strategy to improve neuromuscular function and cardiorespiratory fitness in older adults, which are factors of pivotal importance for the maintenance of functional capacity with aging. However, there is a lack of evidence about the effectiveness of power training (PT) as an alternative to traditional strength training (TST) during CT. Thus, the aim of the present study was to examine the effect of 16 weeks (twice weekly) TST combined with high intensity interval training (TST + HIIT) vs. PT combined with HIIT (PT + HIIT) on functional performance, cardiorespiratory fitness and body composition in older men. Thirty five older men $(65.8 \pm 3.9$ years) were randomly allocated into two training groups: TST + HIIT $(n=18)$, and PT + HIIT $(n=17)$. TST + HIIT performed resistance training at intensities ranging from $65 \%$ to $80 \% 1$ RM at slow controlled speed ( $\cong 2 \mathrm{~s}$ for each concentric phase), whereas PT + HIIT trained at intensities ranging from $40 \%$ to $60 \%$ of $1 \mathrm{RM}$ at maximal intentional speed. Both groups performed HIIT at intensities ranging from 75 to $90 \%$ of $\mathrm{VO}_{2 \text { peak. }}$ Participants performed functional tests (sit-to-stand, timed-up-and-go, stair climbing); cardiopulmonary exercise testing (maximal cycling power output: $\mathrm{W}_{\max }$, peak oxygen uptake: $\mathrm{VO}_{2 \text { peak }}$, cycling economy), as well as body composition assessment (DXA) before, post 8 and post 16 weeks of training. The groups improved similarly $(P<0.05)$ with training in all functional capacity outcomes, $\mathrm{W}_{\max }$, cycling economy, $\mathrm{VO}_{2 \text { peak }}$ and body composition $(\mathrm{P}<0.05)$. These findings suggest that HIIT based CT programs involving TST vs. PT are equally effective in improving functionality, cardiorespiratory fitness and body composition in healthy older men.
\end{abstract}

\section{Introduction}

The World Health Organization (WHO, 2015) public health framework for healthy aging reports three distinct phases for the intrinsic capacity trajectories across the healthy aging lifespan: high and stable capacity, declining capacity and significant loss of capacity (WHO, 2015). Biological aging dramatically impacts functional abilities, such as sit-to-stand, gait, and stair climbing abilities (Reid and Fielding, 2012; Casas-Herrero et al., 2013; Suetta et al., 2019), mainly related to muscle power declines observed during the aging process (Izquierdo et al., 1999; Reid and Fielding, 2012; Alcázar et al., 2020). Together with decreases in functional capacity, aging is also characterized by reductions in lean mass (Suetta et al., 2019) simultaneously to increases in visceral adipose tissue (Garatachea et al., 2015), thus increasing the risk of loss in physical independence and prevalence of cardiovascular disease, respectively. Further, changes in body composition, such as increased body fat and losses in skeletal muscle mass represent important risk factors for the incidence of cardiometabolic diseases such as

\footnotetext{
* Corresponding author at: School of Physical Education, Physiotherapy and Dance, Universidade Federal do Rio Grande do Sul, Rua Felizardo 750, Bairro Jardim Botânico, CEP: 90690-200 Porto Alegre, RS, Brazil.

E-mail address: edu.cadore@ufrgs.br (E.L. Cadore).
} 
hypertension, dyslipidemia, and type II diabetes mellitus in older adults (Sillanpää et al., 2008).

Exercise interventions, in particular concurrent training (CT), a combination of strength and aerobic conditioning is considered a cornerstone to improve neuromuscular and cardiorespiratory functions, body composition indices and functionality in older adults, while simultaneously reducing the cardiometabolic risk profile (Izquierdo et al., 2004; Cadore et al., 2010, 2012; Holviala et al., 2012; Cadore et al., 2018). CT interventions comprising continuous low-to-moderate intensity aerobic training have been associated with improvements in body composition (Sillanpää et al., 2008), improved maximal and submaximal (i.e., movement economy) cardiorespiratory function (Izquierdo et al., 2004; Cadore et al., 2011, 2012), and gains in functional capacity (Wood et al., 2001; Wilhelm et al., 2014). Alternatively, high-intensity interval training (HIIT), characterized by brief and intermittent periods of vigorous activity interspersed with short rest periods, has received growing attention as it shows adaptations that are equivalent to continuous aerobic training; however, in a more timeefficient manner (i.e., exercise performed in a shorter time) (Wisloff et al., 2009; Gibala et al., 2012). Nevertheless, only few studies have investigated the physiological adaptations induced by HIIT in older adults (Knowles et al., 2014; Hwang et al., 2016), and at the best of authors' knowledge, no previous study has investigated the functional and cardiorespiratory adaptations to HIIT as a component of CT in older individuals, which demands for further study.

Muscle power training (PT), characterized by the use of low-tomoderate exercise loads and faster concentric muscle actions $(\leq 1 \mathrm{~s}$ for each concentric phase), may lead to superior improvements in muscle power output and functional abilities when compared to traditional strength training (TST) (Bottaro et al., 2007; Ramirez-Campillo et al., 2014; Straight et al., 2015; Orssatto et al., 2019). Possibly, this advantage occurs because muscle power output seems to be more associated with functional capacity than maximal strength (Reid and Fielding, 2012). Although previous studies have investigated the adaptations induced by PT performed alone in older adults, only few data exist regarding the prescription of PT as a component of CT (Wilhelm et al., 2014). Since HIIT seems to induce greater neuromuscular adaptations when compared to continuous aerobic training (Martinez-Valdes et al., 2018), a question that arises is whether CT interventions composed by PT or TST would promote similar functional adaptations due to its combination with HIIT. In addition, although comparisons between TST versus PT have mainly focused on functional performance and muscle mechanical function (Bottaro et al., 2007; Ramirez-Campillo et al., 2014; Straight et al., 2015; Orssatto et al., 2019), there is a scarce data regarding such comparison on cardiorespiratory fitness and body composition adaptations. In studies investigating younger populations, including cyclists, it has been shown that both TST and PT combined with endurance training promote similar cycling economy and maximal performance gains (for review see Yamamoto et al., 2008). These results suggest that similar cycling economy and maximal performance gains could be observed following TST + HIIT versus PT + HIIT in older adults, which remains speculative. Thus, experimental comparisons between CT programs involving either PT or TST seem warranted.

Based on the above mentioned gaps in the literature, the present study aimed to compare the effects of sixteen weeks of CT involving TST or PT in combination with HIIT on functional performance, cardiorespiratory fitness and body composition in healthy older men. Our initial hypothesis was that PT combined to HIT would promote greater gains on functional capacity, but similar gains in the cardiorespiratory fitness and body composition compared to TST combined with HIIT.

\section{Methods}

\subsection{Study design}

This is a single-center, two-arms, parallel randomized controlled trial
(RCT) with concealed allocation, blinded measurers, and 16 weeks of intervention. Outcomes' assessments were performed pre, post 8 weeks (week 9), and post 16 weeks of training (week 17). The complete screening, recruitment, and allocation of individuals are presented in Fig. 1. The randomization procedure was performed by a researcher not engaged in the recruitment of study participants using a non-commercial public domain web software (www.random.org). Recruitment took place between January and October of 2018 using widely read local newspapers and local University announcements as well as social media. The RCT was part of a larger project, and data on mechanical muscle function outcomes (i.e., maximal strength, rate of force development, maximal power output and muscle thickness) have been published elsewhere (Müller et al., 2020). The primary outcome in this study was the performance in lower limbs functional tests, and the secondary outcomes included maximal cycling power reached during cardiopulmonary exercise testing using a stationary cycle ergometer $\left(W_{\max }\right)$, cycling economy, peak oxygen uptake $\left(\mathrm{VO}_{2 \text { peak }}\right)$, and body composition. The study was conducted according to the Declaration of Helsinki, approved by the local Institutional Ethics Committee (register number 79277917.3.0000.5347) and followed the Consolidated Standards of Reporting Trials (CONSORT) guidelines for non-pharmacological treatment (Boutron et al., 2017).

\subsection{Participants}

Seventy-nine community-dwelling healthy older men volunteered for this study. The participants were carefully informed about the design of the study and special information was provided regarding the possible risks and discomfort related to the procedures. An informed consent form was read and signed prior to study participation. Inclusion criteria included participants older than 60 years old; and participants who were not engaged in any regular and systematic exercise training program in the last 6 months prior to the study starting. Medical evaluations were performed using clinical anamnesis and an effort electrocardiograph (ECG) test to ensure each subject's suitability for the testing procedure. Exclusion criteria included any history of neuromuscular, cognitive, metabolic, hormonal, and cardiovascular diseases (except controlled stage 1 hypertension), as well as smoking or having stopped smoking less than one year prior to the study. In addition, none of the participants were taking any medications that could influence hormonal or neuromuscular metabolism, and all were advised to maintain their normal dietary intake throughout the study period. Moreover, to be included in the pre to post comparisons, participants were required to attend at least $85 \%$ of training sessions. However, participants had the opportunity to recover the training sessions missed in the same week. The sample size was calculated using G POWER software (version 3.0.1), which determined that a sample of $n=20$ participants per group would be necessary to detect an effect size of 0.5 and to provide a statistical power $\geq 85 \%$ for all primary outcomes. In order to assess stability and reliability of the outcome variables, a sub-sample of fourteen participants $(65 \pm 3.8$ years, $85.3 \pm 12.8 \mathrm{~kg}, 172.4 \pm 7.4 \mathrm{~cm}$ ) were assessed twice before the start of intervention to provide control period data (weeks -4 and 0 ). Pre, post 8 (mid-point of intervention) and post 16-weeks of intervention tests were performed by the same investigators, who were blinded regarding groups allocation. The ambient conditions (room temperature: $22-24^{\circ} \mathrm{C}$ ) and time-of-day were kept constant throughout all tests and interventions.

\subsection{Functional capacity}

Functional performance tests comprised sit-to-stand, timed-up-and go (TUG), and stair-climbing. The sit-to-stand test evaluated the highest number of consecutive rising and sitting from a chair in 30s. In the TUG test assessment, participants were instructed to perform the distance as quickly as possible without running. The subjects started sitting, knees and hips flexed at $90^{\circ}$ and with their arms crossed over the chest. At the 


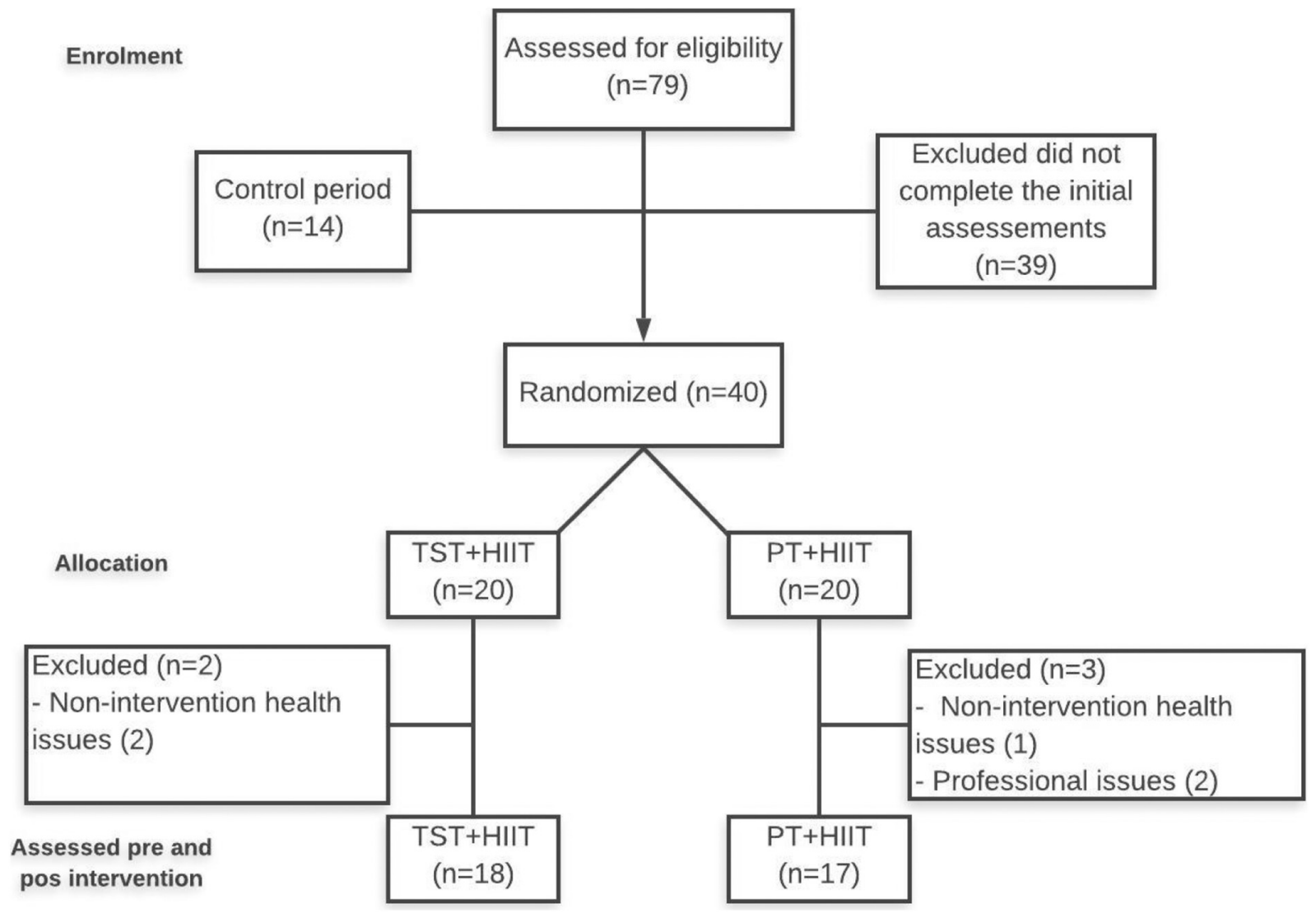

Fig. 1. Flowchart for enrollment, allocation and assessment of individuals. TST + HIIT, concurrent traditional strength and endurance training; PT + HIIT, concurrent power and endurance training.

evaluator's command, the individuals rose from the chair (without using their arms), walked $3 \mathrm{~m}$, walked around a cone, came back and sat back down on the chair. The stair climbing test consisted of climbing a stair with 10 steps (16 cm step height) at a comfortable speed without stopping, and without use of the handrail. Each functional tests was performed three times, and the best value of the three attempts was selected for comparisons.

\subsection{Cardiopulmonary exercise testing}

$\mathrm{VO}_{2 \text { peak }}$ and $\mathrm{W}_{\max }$ were determined using an incremental cardiopulmonary exercise testing on a cycle ergometer (Cybex, USA manufacturer). Firstly, warm-up at $25 \mathrm{~W}$ was performed for $3 \mathrm{~min}$. Subsequently, the load was increased in $25 \mathrm{~W}$ steps every $1 \mathrm{~min}$, while maintaining a cadence of 70-75 rpm, until exhaustion. All the incremental tests were conducted in the presence of a trained physician. The settings of the cycle ergometer were noted to ensure identical set-up at POST testing. The breath-by-breath expired gas was analyzed using a metabolic cart (Quark CPET, Cosmed, Italy). The maximum $\mathrm{VO}_{2}$ value $\left(\mathrm{ml} \cdot \mathrm{kg}^{-1} \cdot \mathrm{min}^{-1}\right)$ obtained close to exhaustion was considered the

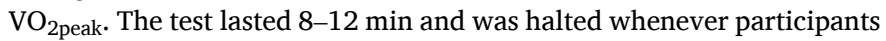
met one of the following criteria: (a) Plateau in oxygen consumption; (b) Heart rate $\geq$ predicted for age; (c) expiratory exchange ratio $>1.15$; (d) subjective perception of effort $>18$ on the 20-point Borg sale, (e) participants being unable to maintain cadence $>70 \mathrm{rpm}$ or when the participant voluntarily requested to interrupt the test. The heart rate was measured continuously using a telemetric heart rate monitor (Polar model 2610, Finland). A telemetry band was positioned to continuously monitor participants' Heart Rate (HR) (Cosmed heart rate monitor integrated in the system). Two blinded and experienced evaluators determined $\mathrm{VO}_{\text {2peak }}$. $\mathrm{W}_{\max }$ was calculated using the equation: $\mathrm{W}_{\max }=$
$\mathrm{W}_{\text {com }}+(\mathrm{t} / 60) \Delta \mathrm{W}$, where $\mathrm{W}_{\text {com }}$ was the Watt loading performed at last fully completed stage, it was the time of the last incomplete stage, and $\Delta \mathrm{W}$ represent the step increment in Watts $(25 \mathrm{~W})$.

\subsection{Cycling economy}

The metabolic cycling economy assessment consisted of performing a steady-state cycle ergometer test using the same metabolic cart used in cardiopulmonary exercise testing. Prior to the test, the subjects remained passively positioned (i.e. at rest) on the cycle ergometer for 2 min to determine basal resting metabolism. After this period, participants performed $10 \mathrm{~min}$ of exercise at a constant load $(75 \mathrm{~W})$ and controlled cadency $(75 \mathrm{rpm})$ for the entire period. In order to analyze the metabolic economy, the first $2 \mathrm{~min}$ and the last $2 \mathrm{~min}$ of the rectangular test were discarded, considering the average oxygen uptake of the central $6 \mathrm{~min}$ of the test.

\subsection{Body composition}

Total and lower limb body composition (total fat mass and fat-free mass) were evaluated using dual-energy X-ray absorptiometry (DXA Hologic Discovery W, USA). During the test, the subjects were positioned on the equipment according to the manufacturer's guidelines: supine, aligned and centered on the examination table with hips and shoulders. The machine was calibrated before each scan according to the manufacturer's specifications. All evaluations were carried out in the morning, $2 \mathrm{~h}$ after the individuals' last meal.

\subsection{Training interventions}

The participants performed both strength training and HIIT in the 
same session, twice weekly, on non-consecutive days, and all groups always performed all strength exercises prior to HIIT, in order to optimize the expected strength/power adaptations (Cadore et al., 2013). Importantly, the different velocity of movement between training interventions occurred only in the bilateral leg press (LP) and bilateral knee extension (KE) exercises performed in isoinertial plate loaded machines. We focused the investigation on those muscles because its importance to the functional activities in older individuals. PT + HIIT group performed the concentric phase of knee extensors exercises at the maximum intentional velocity (hence the term power training), while for safety reasons using a controlled slower velocity in the eccentric movement phase (i.e., approximately $2 \mathrm{~s}$ in the eccentric phase). Conversely, TST + HIT group performed both phases at a slow, controlled velocity (i.e., approximately $2 \mathrm{~s}$ for each phase). The slower muscle actions were controlled using a commercial watch. All participants were oriented and supervised by experienced researchers in order to efficiently perform all exercises at the targeted movement speed. Complete information on all training variables during the period of intervention are shown in Table 2. PT + HIT group performed 3 sets of 8 repetitions at $40 \%$ of $1 \mathrm{RM}$ (weeks 1-4), progressing to 4 sets of 6 repetitions at $60 \%$ of $1 \mathrm{RM}$ (weeks 13-16). The intensities of PT + HIIT intervention were prescribed accordingly to recent recommendations to PT prescription in older individuals (Fragala et al., 2019), since optimal power values are produced approximately in this range of intensities (i. e., from 40 to $60 \%$ of $1-\mathrm{RM}$ ) during isolated knee extensors exercise in older adults (Izquierdo et al., 1999; Strand et al., 2019). TST + HIIT group performed their strength exercise in 2 sets of $12-15$ repetitions at $65 \%$ of $1 \mathrm{RM}$ (weeks $1-4$ ), progressing to 4 sets of $6-8$ repetitions at $80 \%$ of 1 RM (weeks 13-16). The rest interval among successive sets was 180 s. Along with LP and KE exercises, individuals also performed leg curls (isolated knee flexion), chest press, lat pull down, elbow flexion and elbow extension. In these complementary exercises, all participants performed similar training volume compared to the knee extensor exercises (in each respective group), using approximately $60 \%$ of $1 \mathrm{RM}$ in a controlled cadence (i.e. non-explosive). Our purpose was to compare both types of training following each specific recommended training volume and intensities. We did not aim establishing an equal training volume, instead comparing different methods of strength training. The HIIT program was performed using a stationary cycle ergometer. Initially all participants performed 5 min of warm up at $60-65 \%$ of the

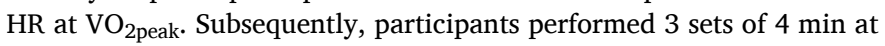
$75-80 \%$ of the $\mathrm{HR}$ at $\mathrm{VO}_{2 \text { peak }}$, (weeks 1-4), progressing until $85-90 \%$ of the $\mathrm{HR}$ at $\mathrm{VO}_{2 \text { peak }}$ (weeks 13-16). While exercising at target intensity, participants were advised to maintain a cadence of 70-75 rpm. The time interval among successive HIIT bouts was $2 \mathrm{~min}$, while biking at a selfselected comfortable speed. Both training groups performed the same HIIT program. All training sessions were carefully supervised by experienced strength and conditioning coaches (minimum 3 concurrently present).

\subsection{Statistical analysis}

SPSS statistical software was used to analyze all data. Normal distribution and homogeneity parameters were checked with Shapiro-Wilk and Levene tests, respectively. Results are reported as mean \pm SD. The training-related effects were assessed using a two-way Analysis of Variance (ANOVA) (group $\times$ time) with Bonferroni's post-hoc testing. If a time vs. group interaction was observed, follow up analysis was proceed using independent $t$-tests for group factor, as well as repeated measures ANOVA for time factor. Significance was accepted when $P<$ 0.05 . The effect size (ES) between pre and post-training for each group was calculated using Cohen's d, represented by the following formula: $\mathrm{ES}=\left(\right.$ Mean $_{\text {post }}-$ Mean $\left._{\text {pre }}\right) / \mathrm{SD}_{\text {pre }}$, which Mean post $_{\text {was }}$ the mean post 16 weeks of training, Mean ${ }_{\text {pre }}$ was the mean pre-training, and $\mathrm{SD}_{\text {pre }}$ is the standard deviation of the pre-training measurements (Nakagawa et al., 2007). The qualitative classification used was ES $>0.20$ (small), ES $>$ 0.50 (moderate), ES $>0.8$ (large), and ES $>1.2$ (very large).

\section{Results}

\subsection{Participants}

In this study, 79 individuals initially volunteered to participate, and 35 older men completed the pre- and post- 16 week measurements and had their data included in the statistical analysis (PT + HIIT: $n=17$, and TST + HIIT: $n=18$ ). Among those who were assessed for eligibility, there were no apparent differences (i.e., between participants who did not complete or even initiate the assessments with the participants who completed the study). The reasons for not participating included personal and professional issues not detailed by the participants. No participant mentioned any concerns or disagreements regarding the interventions of the study. Physical characteristics are shown in Table 1.

\subsection{Control period, physical characteristics and adverse effects}

Data obtained during the control period are presented in Supplementary file. During the control period (i.e., between week -4 and week 0 ), no significant changes were observed for any outcome parameters. Before and after training, there were no differences between the groups in body mass $(\mathrm{kg})$, height $(\mathrm{cm})$, age (years) Table 1 . All individuals who entered the pre and post analysis attended at $100 \%$ of training sessions. During the intervention period, no adverse effects were reported related to the training programs.

\subsection{Overall findings}

At baseline, there were no differences between the group for any of the outcomes assessed (i.e., sit-to-stand, climbing stairs, TUG, body composition, cycling economy, $\mathrm{W}_{\max }$, and $\mathrm{VO}_{2 \text { peak }}$ ). In addition, after 16 weeks of training, there were no significant time vs. group interaction or significant group effect for any of the variables $(P>0.05)$.

\subsection{Functional capacity}

A significant time effect $(P<0.001)$ was observed for the sit-to-stand test with both PT + HIIT and TST + HIIT demonstrating increased values after 8 weeks ( $14.3 \pm 30.0 \%$ and $17.2 \pm 13.3 \%$, respectively, $P<0.001)$, and 16 weeks intervention (PT + HIIT: $37.3 \pm 27.1 \%$, ES $=1.46$; and

Table 1

Physical characteristics pre and post intervention.

\begin{tabular}{|c|c|c|c|c|c|c|}
\hline & & $\mathrm{PT}+\operatorname{HIIT}(n=17)$ & & & TST + HIIT $(n=18)$ & \\
\hline & Pre & Post 8 & Post 16 & Pre & Post 8 & Post 16 \\
\hline Age (years) & $64.3 \pm 3.3$ & $64.3 \pm 3.3$ & $65.1 \pm 3.3$ & $65.7 \pm 4.2$ & $65.7 \pm 4.2$ & $66.4 \pm 4.3$ \\
\hline Height (cm) & $176 \pm 7.2$ & $176 \pm 7.2$ & $176 \pm 7.2$ & $174.2 \pm 2.2$ & $174.2 \pm 2.2$ & $174.2 \pm 2.2$ \\
\hline Body mass (kg) & $84.7 \pm 14.8$ & $84.9 \pm 14.4$ & $85.2 \pm 14.4$ & $89.9 \pm 9.1$ & $90.1 \pm 9.6$ & $89.4 \pm 9.1$ \\
\hline
\end{tabular}

TST, traditional strength; PT, power training; HIIT, high-intensity interval training. 
Table 2

Training periodization.

\begin{tabular}{|c|c|c|c|c|c|c|c|c|}
\hline \multicolumn{4}{|l|}{ TST } & \multicolumn{3}{|l|}{$\mathrm{PT}$} & \multicolumn{2}{|l|}{ HIIT } \\
\hline Week & Sets & Repetitions & Intensity (\% 1RM) & Sets & Repetitions & Intensity (\% 1RM) & Time (min) & Intensity $\left(\% \mathrm{VO}_{2 \text { peak }}\right)$ \\
\hline $1-4$ & 2 & $12-15$ & 65 & 3 & 8 & 40 & $3 \times 4 / 2$ & $75-80$ \\
\hline $5-8$ & 3 & $10-12$ & 70 & 3 & 8 & 50 & $3 \times 4 / 2$ & $80-85$ \\
\hline $9-12$ & 3 & $8-10$ & 75 & 4 & 6 & 55 & $4 \times 4 / 2$ & $80-85$ \\
\hline $13-16$ & 4 & $6-8$ & 80 & 4 & 6 & 60 & $4 \times 4 / 2$ & $85-90$ \\
\hline
\end{tabular}

TST, traditional strength training; PT, power training; HIIT, high-intensity interval training.

Table 3

Data expressed as percentual and absolute values (mean \pm standard deviation) of body composition, cycling economy and cardiorespiratory fitness.

\begin{tabular}{|c|c|c|c|c|}
\hline Outcomes & Group & Pre & Post 8 & Post 16 \\
\hline \multirow[t]{2}{*}{ Total body mass (Kg) } & PT + HIIT & $84.7 \pm 14.8$ & $84.9 \pm 14.4$ & $85.2 \pm 14.4$ \\
\hline & TST + HIIT & $89.3 \pm 9.9$ & $90.1 \pm 9.6$ & $89.4 \pm 9.1$ \\
\hline \multirow[t]{2}{*}{ Absolute Body fat (Kg) } & PT + HIIT & $26.8 \pm 8.4$ & $26.3 \pm 8.0$ & $26.5 \pm 7.6$ \\
\hline & TST + HIIT & $29.5 \pm 5.5$ & $29.4 \pm 5.6$ & $28.8 \pm 5.3$ \\
\hline \multirow[t]{2}{*}{ Absolute fat-free mass (kg) } & PT + HIIT & $54.7 \pm 6.8$ & $55.4 \pm 6.9^{*}$ & $55.5 \pm 7.3^{*}$ \\
\hline & TST + HIIT & $57.1 \pm 6.6$ & $57.3 \pm 6.3^{*}$ & $57.2 \pm 6.5^{\star}$ \\
\hline \multirow[t]{2}{*}{ Relative body fat (\%) } & PT + HIIT & $31.1 \pm 4.5$ & $30.5 \pm 4.5^{*}$ & $30.7 \pm 4.1 *$ \\
\hline & TST + HIIT & $32.7 \pm 4.3$ & $32.5 \pm 4.5^{\star}$ & $32.2 \pm 4.7^{\star}$ \\
\hline \multirow[t]{2}{*}{ Relative fat-free mass (\%) } & $\mathrm{PT}+\mathrm{HIIT}$ & $65.1 \pm 4.3$ & $65.7 \pm 4.2^{*}$ & $65.6 \pm 3.9^{*}$ \\
\hline & TST + HIIT & $63.5 \pm 3.9$ & $63.7 \pm 4.0^{*}$ & $64.1 \pm 4.3^{*}$ \\
\hline \multirow[t]{2}{*}{ Cycling economy $\left(\mathrm{mL} \cdot \mathrm{kg}^{-1} \cdot \mathrm{min}^{-1}\right)$} & PT + HIIT & $15.0 \pm 3.1$ & $14.2 \pm 2.7^{*}$ & $13.9 \pm 2.7^{* *}$ \\
\hline & TST + HIIT & $15.0 \pm 1.8$ & $13.8 \pm 2.0^{*}$ & $13.8 \pm 2.3^{* *}$ \\
\hline \multirow[t]{2}{*}{$\mathrm{W}_{\text {Max }}$ (watts) } & PT + HIIT & $196.3 \pm 17.1$ & $206.0 \pm 24.2^{*}$ & $219.9 \pm 32.2^{* *}$ \\
\hline & TST + HIIT & $194.6 \pm 26.4$ & $205.5 \pm 32.2^{*}$ & $216.3 \pm 28.2^{* * *}$ \\
\hline \multirow[t]{2}{*}{$\mathrm{VO}_{2 \text { peak }}\left(\mathrm{mL} \cdot \mathrm{kg}^{-1} \cdot \mathrm{min}^{-1}\right)$} & $\mathrm{PT}+\mathrm{HIIT}$ & $24.5 \pm 6.1$ & $26.2 \pm 4.3^{*}$ & $27.8 \pm 5.2^{* * *}$ \\
\hline & TST + HIIT & $24.8 \pm 3.8$ & $25.7 \pm 4.4^{*}$ & $26.8 \pm 4.5^{* * *}$ \\
\hline
\end{tabular}

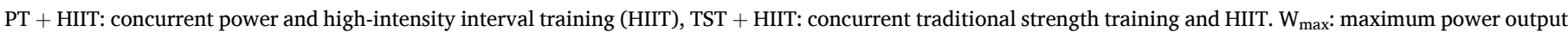
at cycle ergometer; $\mathrm{VO}_{2 \mathrm{max}}$ : peak oxygen consumption. Significant differences between pre and post interventions.

$$
\begin{aligned}
& { }_{* *}^{*} \mathrm{P}<0.05 . \\
& { }_{* * *} \mathrm{P}<0.01 .
\end{aligned}
$$

TST + HIIT: $29.5 \pm 15.9 \%, E S=0.91, \mathrm{P}<0.001)$. The values obtained at 16 weeks were significant greater than those observed after 8 weeks $(P$ $<0.001)$. In addition, there was a time effect for TUG $(P<0.01)$ with both PT + HIIT and TST + HIIT improving after 16 weeks of intervention (PT + HIIT: $-6.5 \pm 14.0 \%$, ES $=-0.54$; and, TST + HIIT: $-7.1 \pm 10.4 \%$, $\mathrm{ES}=-0.47, P<0.001$ ). Values at 16 weeks were improved (lower) than at post $8 \mathrm{wk}$. $(\mathrm{P}<0.01)$, while no difference between pre and post 8 weeks was observed. Likewise, a time effect $(P<0.001)$ was observed for the stair climbing test as well. Both PT + HIIT and TST + HIIT improved this outcome after 8 weeks $(-5.7 \pm 13.9 \%$ and $-3.9 \pm 13.2 \%$, respectively, $P<0.05$ ) and 16 weeks (PT + HIIT: $-10.8 \pm 12.3 \%$, ES $=$ -0.78 ; and TST + HIIT: $-8.8 \pm 15.2 \%$, ES $=-0.54, \mathrm{P}<0.001)$, with better performance after 16 weeks compared with 8 weeks $(\mathrm{P}<0.01)$. The results of the functional tests are presented in Fig. 2.

\subsection{Cardiopulmonary fitness and cycling economy}

A significant time effect was noted for $\mathrm{W}_{\max }(\mathrm{P}<0.001)$, with both PT + HIIT and TST + HIIT increasing this variable after 8 weeks $(4.9 \pm$ $8.0 \%$ and $5.4 \pm 6.7 \%$, respectively, $\mathrm{P}<0.001)$ as well as after 16 weeks of training (PT + HIIT: $11.7 \pm 11.2 \%$, ES: 1.17 , TST + HIIT: $11.6 \pm$ $9.9 \%$, ES $=0.75 ; \mathrm{P}<0.001$ ), with no differences between groups (Table 3). In addition, a time effect was observed for $\mathrm{VO}_{2 \text { peak }}(P<0.05)$, with both groups increasing their values at 8 weeks $(11.1 \pm 22.3 \%$, and $3.9 \pm 7.8 \%$, respectively, $P<0.05$ ), and 16 weeks of training (PT + HIIT: $19.0 \pm 37.8 \%$, ES $=0.54$; TST + HIIT: $8.6 \pm 10 \%$, ES $=0.53, \mathrm{P}<0.01$ ) with no differences between groups (Table 3 ). Both PT + HIIT and TST + HIIT improved their cycling economy after 8 weeks (PT + HIIT: -4.7 $\pm 10.7 \%$, TST + HIIT: $-7.6 \pm 9.9 \% ; \mathrm{P}<0.001)$, and 16 weeks $(\mathrm{PT}+$ HIIT:-5.7 \pm 17.2\%, ES:-0.34; TST + HIIT:-7.5 \pm 9.5\%, ES:-0.60; P < a)

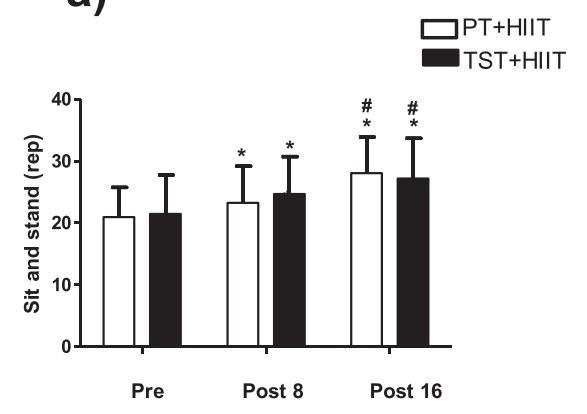

b)

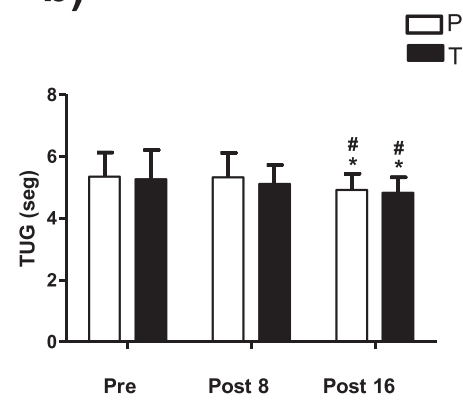

c)

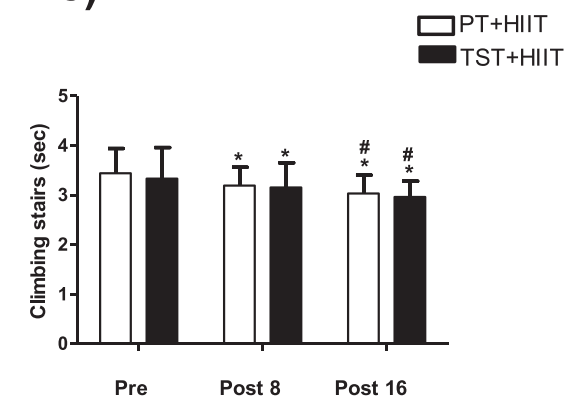

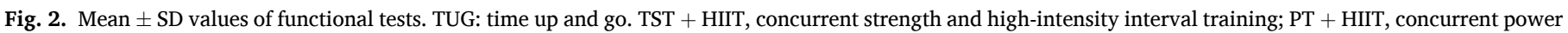
and high-intensity interval training. ${ }^{*}$ Significantly greater than the pre-training $(P<0.001)$; \#Significantly greater than the post $8(P<0.001)$. 
$0.001)$ with no differences between groups. No difference was observed in the post 16 compared with post 8 for this variable (Table 3 ).

\subsection{Body composition}

All body composition variables are described in Table 3. A time effect ( $\mathrm{P}<0.05)$ was observed for body fat percentage, with both PT + HIIT and TST + HIIT decreasing this parameter after 8 and 16 weeks (PT + HIIT: $-1.8 \pm 2.6 \%$, ES: -0.09 ; TST + HIIT: $-0.5 \pm 3.9 \%$; ES: -0.12 ). There were no differences between groups in body fat percentage, as well as no differences between post 8 compared to post 16 values. In addition, a time effect was noted for fat-free mass percentage $(\mathrm{P}<0.05)$ with both PT + HIIT and TST + HIIT increasing these values after 8 and 16 weeks (PT + HIIT: $0.9 \pm 1.2 \%$, ES $=0.10$; and, TST + HIIT: $0.2 \pm$ $2.0 \%, \mathrm{ES}=0.13, \mathrm{P}<0.05)$. No difference between groups as well as no differences between 8 and 16 weeks were observed. No changes were observed in absolute body fat $(P=0.10)$ and total body mass after the training period. In terms of absolute fat-free mass, a time effect $(\mathrm{P}<$ 0.05) was observed in both PT + HIIT and TST + HIIT after 8 and 16 weeks intervention (PT + HIIT: $1.2 \pm 2.0 \%$, ES $=0.11$; and, TST + HIIT: $0.5 \pm 2.2 \%$, ES $=0.02, \mathrm{P}<0.05$ ) with no difference observed between groups, as well as no differences between 8 and 16 weeks.

\section{Discussion}

The main finding from the present study was that concurrent training protocols, combining either traditional strength or power training with HIIT, led to similar improvements in functional capacity in healthy older men, along with similar gains in $\mathrm{W}_{\mathrm{max}}$, cycling economy, $\mathrm{VO}_{2 \text { peak }}$ and body composition. These findings are relevant as they demonstrate that concurrent training programs employing power vs. traditional strength training may be equally effective inducing functional and physiological adaptations in older adults.

One of the paramount goals of exercise interventions across the aging is to maintain individual independency to perform various daily living activities (Studenski et al., 2011; Izquierdo and Cadore, 2014; Cadore and Izquierdo, 2018). In the present study, sit-to-stand ability improved by $14-17 \%$ after 8 weeks concurrent training, to reach a $30 \%$ improvement after 16 weeks of intervention. Likewise, marked gains in TUG and stair-climbing performance were observed after 16 weeks of concurrent training (i.e., approximately $+7 \%$ and $+11 \%$, respectively). These findings are in agreement with previous study reports investigating the translational benefits of strength training (Sayers et al., 2007; Bottaro et al., 2007; Bean et al., 2002; Ramirez-Campillo et al., 2014; BeltranValls et al., 2014) or concurrent training (Wood et al., 2001; Wilhelm et al., 2014; Cadore et al., 2018; Teodoro et al., 2019) on functional capacity in older populations, showing similar magnitudes of improvements produced by the two exercise paradigms.

Some studies have reported greater improvements in selected functional abilities following power training in older adults when compared to traditional strength training (Bottaro et al., 2007; Correa et al., 2012; Ramirez-Campillo et al., 2014) although not always a consistent finding (Seynnes et al., 2008). In a recent meta-analysis by Orssatto et al. (2019), it was concluded that the use of maximal intend velocity during strength training (i.e., PT) resulted in greater improvements than TST in the combined functional tests, Short Physical Performance Battery (SPPB), whereas similar enhancements were observed for TUG test, 30-s chair stand test, 5-times chair stand test, stair climbing test, short as well long walking tests. In the present study, the absence of measurable differences between the intervention groups in terms of functional capacity improvements may have occurred due to the HIIT element, which was a common feature in both training programs. In addition, in the present cohort of healthy old males we previously observed an advantage of PT + HIIT compared to TST + HIIT for the training-induced improvement in explosive force outcomes (Müller et al., 2020). In contrast, maximal muscle strength and power output as well as morphological outcomes such as muscle thickness along with endurance performance was found to improve similarly between groups (Müller et al., 2020). Consequently, given that PT + HIIT and TST + HIIT led to similar improvements in a majority of variables related to mechanical muscle function, it is of no surprise that these different training regimes led to similar improvements in functional capacity in the present cohort of old male adults.

Both training interventions led to substantial (and comparable) effects on $\mathrm{W}_{\text {max }}$ and $\mathrm{VO}_{2 \text { peak }}$ after 8 and 16 weeks intervention. In addition, improvements in cycling economy were noted in both TST + HIT and PT + HIIT after 8 and 16 weeks of training. It is likely that these adaptations were mainly the result of the HIIT training performed, which was the same in both groups. On the other hand, it has been shown that strength training per se may enhance cycling economy and improve short-term cycling performance (i.e., $\mathrm{W}_{\max }$, time to exhaustion) both in untrained individuals (Hansen et al., 2007; Loveless et al., 2005) as well as in high-level trained cycling athletes (Bastiaans et al., 2001; Rønnestadand et al., 2013). The absence of differences between groups agrees with a systematic review by Yamamoto et al. (2008) since these authors observed no differences between power training versus maximal strength training on the improvement in the cycling economy and performance in trained cyclists. Regardless, the present improvement in cycling economy is likely to have important clinical implications, because older adults that are metabolically more efficient during given activities of daily living may present lower cardiovascular responses to a given effort. Consequently, tasks that involve walking, sit-to-stand, load carrying and stair climbing may be performed at a lower metabolic cost (Hartman et al., 2007), hence potentially resulting in an increased endurance capacity for these specific tasks. Along with oxidative myocellular adaptations induced by HIIT (Gibala et al., 2012), the improvements in maximal muscle strength and explosive force capacity (RFD) following TST + HIIT and PT + HIIT (Müller et al., 2020) may result in a reduced need for type II muscle fiber recruitment at given submaximal loadings, potentially inducing lower metabolic cost (Izquierdo et al., 2003). In support of this hypothesis, neuromuscular activation (i.e., normalized electromyographic signal) was reduced in older adults at different submaximal loadings during submaximal ergometer cycling following a period of concurrent training, suggesting a reduction in the relative loading intensity (reduced $\%$ of max) (Cadore et al., 2011).

Only small albeit statistically significant improvements in body composition were observed in the present study, such as a reduction in the body fat percentage and an increase in the fat-free mass percentage and absolute fat-free mass. These changes were lower than reported by Sillanpää et al. (2008), who demonstrated improvements ranging from 5 to $8 \%$ in the body composition outcomes following 21 weeks of strength, endurance and concurrent training in older men (Sillanpää et al., 2008), although more similar to the findings by Sillanpää et al. (2009) investigating the physiological effects induced by 21 weeks of strength, endurance or concurrent training in middle-aged and older women. In addition, it has been also demonstrated that body composition outcomes were unaffected following a HIIT intervention in healthy older adults (Hwang et al., 2016). The absence of greater changes in the body composition outcomes in the present study could be due to the absence of caloric ingestion control. Although we advised all participants to maintain their normal pattern of dietary intake throughout the study, a constancy in this parameter was difficult to control. In addition, concurrent training sessions were performed twice weekly, which might represent an insufficient quantity of exercise training (e.g. too low volume) to induce marked changes in body composition despite the 16 week-period of training (Jonh et al., 2001). Although only minor in magnitude, however, the present decreases in the body fat percentage and increases in the fat-free mass may be considered as important physiological adaptations, since even small changes in body composition have been associated with improved health index and decreased cardiometabolic risk (Ismail et al., 2012). 
A number of limitations may be mentioned for the present study. The present study sample consisted of men only, thus limiting the generalization of the findings to the female population. In addition, the absence of dietary intake control limits interpretation of the present changes in body composition. Besides, the participants performed two specific concurrent training protocols that were not matched by total overload, which could influence the body composition adaptations. Moreover, we focused our comparison specifically in the lower limbs, and therefore, it is unclear if the same results would be observed in other muscle groups. Nevertheless, hip and knee extensors are the main muscle groups related to functional capacity in older individuals, so our results have important clinical application. As an another strength, this study is the first to examine the adaptations in functional capacity, body composition, cycling economy, and cardiorespiratory fitness following concurrent HIIT training involving either power training or traditional strength training in older men. The significant improvements in functional capacity, cycling economy and cardiorespiratory fitness observed following 16 weeks of concurrent training underlines the translational value of the present study.

\section{Conclusions}

The main finding of the present study was that 16 weeks of HIIT combined with power or traditional strength training promoted similar gains in functional capacity, cycling economy, $\mathrm{W}_{\max }, \mathrm{VO}_{2 \text { peak, }}$ and body composition (increased lean mass, reduced fat mass) in healthy older men. Thus, when combined with concurrent within-session HIIT, power training composed by low volume of repetitions per set (6-8) performed at moderate loading intensities (40-60\% of $1-\mathrm{RM}$ ) appears equally as effective as traditional strength training involving greater training volume and intensity for improving functional capacity, cardiorespiratory fitness and body composition in older men.

Supplementary data to this article can be found online at https://doi. org/10.1016/j. exger.2021.111321.

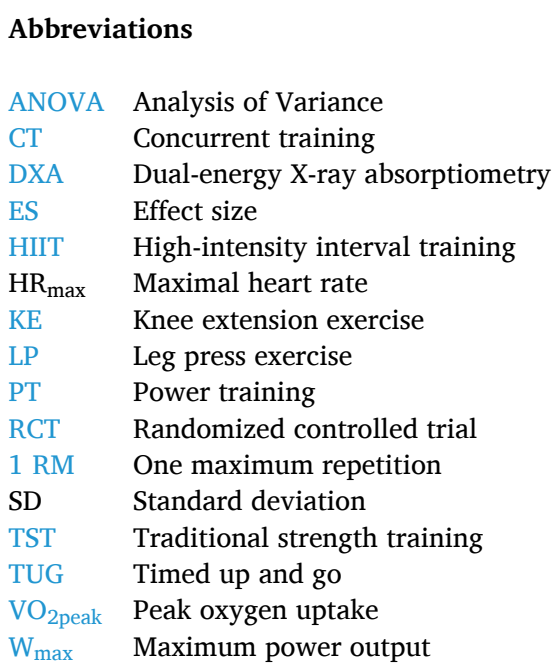

\section{Declaration of competing interest}

The authors declare that they have no known competing financial interests or personal relationships that could have appeared to influence the work reported in this paper.

\section{Acknowledgments}

The authors thank to National Council for Scientific and Technological Development (CNPq, Brazil) and Coordination for the Improvement of Higher Education Personnel (CAPES, Brazil) for its support to this project. In addition, we also gratefully acknowledge to all the participants who participated in this research and made this project possible.

\section{References}

Alcázar, J., et al., 2020. Age-and sex-specific changes in lower-limb muscle power throughout the lifespan. J Gerontol Med Sci 75, 1369-1378. https://doi.org/ 10.1093/gerona/glaa013.

Bastiaans, J.J., et al., 2001. The effects of replacing a portion of endurance training and by explosive strength training on performance in trained cyclist. Eur. J. Appl. Physiol. (86), 79-84. https://doi.org/10.1007/s004210100507.

Bean, J.F., et al., 2002. The relationship between leg power and physical performance in mobility-limited older people. J. Am. Geriatr. Soc. 50 (3), 461-467. https://doi.org/ 10.1046/j.1532-5415.2002.50111.x.

BeltranValls, M.R., et al., 2014. Explosive type of moderate-resistance training induces functional, cardiovascular, and molecular adaptations in the elderly. AGE 36, 759-772. https://doi.org/10.1007/s11357-013-9584-1.

Bottaro, M., et al., 2007. Effect of high versus low-velocity resistance training on muscular fitness and functional performance in older men. Eur. J. Appl. Physiol. 99, 257-264. https://doi.org/10.1007/s00421-006-0343-1.

Boutron, I., et al., 2017. CONSORT statement for randomized trials of nonpharmacologic treatments: a 2017 update and a CONSORT extension for nonpharmacologic trial abstracts. Ann. Intern. Med. 167 (1), 40-47. https://doi.org/10.7326/M17-0046.

Cadore, E.L., Izquierdo, M., 2018. Muscle power training: a Hallmark for muscle function retaining in frail clinical setting. JAMDA 19, 190-192. https://doi.org/10.1016/j. jamda.2017.12.010.

Cadore, E.L., et al., 2010. Physiological effects of concurrent training in elderly men. Int. J. Sports Med. 31, 689-697. https://doi.org/10.1055/s-0030-1261895.

Cadore, E.L., et al., 2011. Neuromuscular economy, strength and endurance in healthy elderly men. J Strength Cond Res. (25), 997-1003. https://doi.org/10.1519/ JSC.0b013e3181d650ba.

Cadore, E.L., et al., 2012. Strength prior to endurance intra-session exercise sequence optimizes neuromuscular and cardiovascular gains in elderly men. Exp. Gerontol. 47, 164-169. https://doi.org/10.1016/j.exger.2011.11.013.

Cadore, E.L., et al., 2013. Neuromuscular adaptations to concurrent training in theelderly: effects of intra session exercise sequence. Age 35, 891-903. https://doi. org /10.1007/s11357-012-9405-y.

Cadore, E.L., et al., 2018. Functional and physiological adaptations following concurrent training using sets with and without concentric failure in elderly men: a randomized clinical trials. Expermental Gerontology. Ver. (110), 182-190. https://doi.org/ 10.1016/j.exger.2018.06.011.

Casas-Herrero, A., et al., 2013. Functional capacity, muscle fat infiltration, power output and cognitive impairment in institutionalized frail oldest-old. Rejuvenation Res. (16), 396-403. https://doi.org/10.1089/rej.2013.1438.

Correa, C.S., et al., 2012. 3 diff erent types of strength training in older women. Int. J. Sports Med. 33, 962-969. https://doi.org/10.1055/s-0032-1312648.

Fragala, M.S., et al., 2019. Resistance training for older adults: position statement from the national strength and conditioning association. Journal of Strength and Conditioning Research 33 (8), 2019-2052. https://doi.org/10.1519/ JSC.0000000000003230.

Garatachea, N., et al., 2015. Exercise Attenuates the Major Hallmarks of Aging. REJ2014-1623. https://doi.org/10.1089/rej.2014.1623.

Gibala, M.J., et al., 2012. Physiological adaptations to low-volume, high-intensity interval training in health and disease. Physioly 590 (5), 1077-1084. https://doi. org/10.1113/jphysiol.2011.224725.

Hansen, E.A., et al., 2007. Strength training reduces freely chosen pedal rate during submaximal cycling. Eur. J. Appl. Physiol. 101, 419-426. https://doi.org/10.1007/ s00421-007-0515-7.

Hartman, M.J., et al., 2007. Resistance training improves metabolic economy during functional tasks in older adults. J Strength Cond Res 21, 91-95. https://doi.org/ 10.1519/00124278-200702000-00017.

Holviala, J., et al., 2012. Effects of strength, endurance and combined training on muscle strength, walking speed and dynamic balance in aging men. Eur. J. Appl. Physiol. 112, 1335-1347. https://doi.org/10.1007/s00421-011-2089-7.

Hwang, C.L., et al., 2016. Novel all-extremity high-intensity interval training improves aerobic fitness, cardiac function and insulin resistance in healthy older adults. Exp Gerontology 82, 112-119. https://doi.org/10.1016/j.exger.2016.06.009.

Ismail, I., et al., 2012. A systematic review and meta-analysis of the effect of aerobic vs. resistance exercise training on visceral fat. Obes. Rev. 13, 68-91. https://doi.org/ 10.1111/j.1467-789X.2011.00931.x.

Izquierdo, M., Cadore, E.L., 2014. Muscle power training in the institutionalized frail: a new approach to counteracting functional declines and very late-life disability. Current Medical Research \& Opinion 1-6. https://doi.org/10.1185/ 03007995.2014.908175.

Izquierdo, M., et al., 1999. Maximal strength and power characteristics in isometric and dynamic actions of the upper and lower extremities in middle-aged and older men. Acta Physiol. Scand. 167, 57-68. https://doi.org/10.1046/j.1365-201x.1999.00590. $\mathrm{x}$.

Izquierdo, M., et al., 2003. Effects of strength training on submaximal and maximal endurance performance capacity in middle-aged and older men. Journal of Strength and Conditioning Research 17 (1), 129-139. https://doi.org/10.1519/1533-4287 (2003) $017<0129$ : eostos $>2.0$. co; 2 .

Izquierdo, M., et al., 2004. Once weekly combined resistance and cardiovascular training in health yolder men. Med. Sci. Sports Exerc. 36, 435-443. https://doi.org/10.1249/ 01.MSS.0000117897.55226.9A. 
Jonh, M., et al., 2001. Appropriate intervention strategies for weight loss and prevention of weight regain for adults. Official Journal of the American College of Sports Medicine. https://doi.org/10.1097/00005768-200112000-00026.

Knowles, A., et al., 2014. Impact of low-volume, high-intensity interval training on maximal aerobic capacity, health-related quality of life and motivation to exercise in ageing men. AGE 25-37. https://doi.org/10.1007/s11357-015-9763-3.

Loveless, D., et al., 2005. Maximal leg-strength training improves cycling economy in previously untrained men. Med. Sci. Sports Exerc. (37), 1231-1236. https://doi.org/ 10.1249/01.mss.0000170071.33880.f8.

Martinez-Valdes, E., et al., 2018. Motor unit conduction velocity changes to HIIT versus continuous training. Med. Sci. Sports Exerc. (50) 2339-2350. https://doi.org/ 10.1249/MSS.0000000000001705.

Müller, D.C., et al., 2020. Adaptations in mechanical muscle function, muscle morphology, and aerobic power to high-intensity endurance training combined with either traditional or power strength training in older adults: a randomized clinical trial. Eur J Appl Physiol. May. (5) 1165-1177. https://doi.org/10.1007/s00421020-04355-z.

Nakagawa, S., et al., 2007. Effect size, confidence interval and statistical significance: a practical guide for biologists. Biol. Rev. Camb. Philos. Soc. 82 (4), 591-605. https:// doi.org/10.1111/j.1469-185X.2007.00027.x.

Orssatto, L., et al., 2019. Effects of resistance training concentric velocity on older adults' functional capacity: a systematic review and meta-analysis of randomised trials. Exp gerontology 127, 110731. https://doi.org/10.1016/j.exger.2019.110731.

Ramirez-Campillo, R., et al., 2014. High-speed resistance training is more effective than low-speed resistance training to increase functional capacity and muscle performance in older women. Exp. Gerontology. 58, 51-57. https://doi.org/ 10.1016/j.exger.2014.07.001.

Reid, K.F., Fielding, R.A., 2012. Skeletal muscle power and functioning in older adults. Exerc. Sport Sci. (40), 1-12. https://doi.org/10.1097/JES.0b013e31823b5f13.

Rønnestadand, B.R., et al., 2013. Optimizing strength training for running and cycling endurance performance: a review. Scand. J. Med. Sci. Sports 1-10. https://doi.org/ $10.1111 /$ sms.12104.

Sayers, S.P., et al., 2007. High-speed power training: a novel approach to resistance training in older men and women. A brief review and pilot study. J Strength Cond Res (2), 518-526. https://doi.org/10.1519/R-20546.1.

Seynnes, O., et al., 2008. Physiological and functional responses to low-moderate versus high-intensity progressive resistance training in frail elders. J. Gerontol. A Biol. Sci. Med. Sci. 2004 (59), 503-509. https://doi.org/10.1093/gerona/59.5.M503.
Sillanpää, E., et al., 2008. Body composition and fitness during strength and/or endurance training in older men. Med. Sci. Sports Exerc. 40, 950-958. https://doi. org/10.1249/MSS.0b013e318165c854.

Sillanpää, E., et al., 2009. Body composition, fitness, and metabolic health during strength and endurance training and their combination in middle-aged and older women. Eur. J. Appl. Physiol. 106, 285-296. https://doi.org/10.1007/s00421-0091013-x.

Straight, C.R., et al., 2015. Effects of resistance training on lower-extremity muscle power in middle-aged and older adults: a systematic review and meta-analysis of randomized controlled trials. Sports Med. (46), 353-364. https://doi.org/10.1007/ s40279-015-0418-4.

Strand, K.L., et al., 2019. Optimal loads for power in older men and women using plateloaded resistance machines. Exp. Gerontol. 124, 110638 https://doi.org/10.1016/j. exger.2019.110638.

Studenski, S., et al., 2011. Gait speed and survival in older adults. JAMA 305, 50-58. https://doi.org/10.1001/jama.2010.1923.

Suetta, C., et al., 2019. The Copenhagen sarcopenia study: lean mass, strength, power and physical function in a Danish cohort aged 20-93 years. J. Cachexia. Sarcopenia Muscle 10, 1316-1329. https://doi.org/10.1002/jcsm.12477.

Teodoro, J., et al., 2019. Concurrent training performed with and without repetitions to failure in older men: a randomized clinical trial. Scand. J. Med. Sci. Sports (00), 1-12. https://doi.org/10.1111/sms.13451.

WHO, 2015. In: Beard, J., Cassels, A. (Eds.), World Report on Ageing and Health. World Health Organization, Geneva, Switzerland. https://doi.org/10.1016/S0140-6736 (15)00516-4.

Wilhelm, E.N., et al., 2014. Concurrent strength and endurance training exercise sequence does not affect neuromuscular adaptations in older men. Exp. Gerontol. 60, 207-214. https://doi.org/10.1016/j.exger.2014.11.007.

Wisloff, U., et al., 2009. High-intensity interval training to maximize cardiac benefits of exercise training? American College of Sports Medicine 139-146. https://doi.org/ 10.1097/JES.0b013e3181aa65fc.

Wood, R.H., et al., 2001. Concurrent cardiovascular and resistance training in health yolder adults. Med. Sci. Sports Exerc. 33, 1751-1758. https://doi.org/10.1097/ 00005768-200110000-00021.

Yamamoto, L.M., et al., 2008. The effects of resistance training on road cycling performance among highly trained cyclists: a systematic review. Journal of Strength and Conditioning Research 24 (2), 560-566. https://doi.org/10.1519/ JSC.0b013e3181c86583. 